\title{
From 'Designated Member' back to 'Full Committee'?
}

Sean Smith and his wife Liz were both researchers at Great Eastern University. Sean, who was a member of the IACUC, was quite disturbed about an ongoing protocol review that he believed compromised the well-being of some sheep. The IACUC chairman had appointed the attending veterinarian (AV) as the sole Designated Member Reviewer for a protocol that Smith assumed would be a 'no brainer' for a veterinarian. The Principal Investigator (PI) proposed a surgical procedure utilizing appropriate anesthesia but no analgesia. Smith had done similar surgery some years earlier and acted as a consultant for the PI. He specifically reminded the PI to use an analgesic, but the PI chose not to, claiming that because the sheep were expected to be up and walking within an hour after surgery, analgesia was not needed. Smith was positive that the AV would notice this and request that an analgesic be added before the protocol could be approved.
But, when Smith asked the PI about the progress of the protocol, the latter said that he needed to respond to some small items in order for the protocol to be approved, but that the use of analgesia was not one of them. That evening, Sean related the story to Liz, and as they mulled the problem over, Liz came up with an idea. "Sean," she said, "Why don't you just ask for Full Committee Review [FCR] before the protocol is approved by the veterinarian?" "I can't," he answered, "I blew my chance when the IACUC office asked the committee members if anybody wanted FCR and nobody said that they did." But Liz was not about to give up. She read through all of the IACUC material that Sean brought home and found a passage from an Office of Laboratory Animal Welfare Notice ${ }^{1}$ stating in part that "...any member of the IACUC may, at any time, request to see the revised protocol and/or request FCR of the protocol." "Doesn't that count, Sean?" she said. "Not really," he responded, "that's only for special circumstances." "Well," said Liz, "I didn't see anything in what I read which suggested that an IACUC member can't request a FCR before the Designated Member Reviewers have come to a decision. I think you should ask for a FCR as soon as you can, like sending an e-mail right now to the IACUC office."

Is Liz Smith right? Can an IACUC member who is not a Designated Member Reviewer request a Full Committee Review while the Designated Member Reviewers are still deliberating?

1. Office of Laboratory Animal Welfare. Guidance to IACUCs Regarding Use of Designated Member Review (DMR) for Animal Study Proposal Review Subsequent to Full Committee Review (FCR). Notice NOT-OD-09-035. (National Institutes of Health, Washington, DC, 8 January 2009). $<$ http://grants.nih.gov/grants/guide/noticefiles/NOT-OD-09-035.html>

\section{RESPONSE}

\section{Not too late for FCR}

\section{Sridhar Samineni, DVM, MS \& Richard W. Ermel, DVM, MPVM, PhD, DACLAM}

The Animal Welfare Act and Regulations (AWARs) ${ }^{1}$ and PHS Policy on Humane Care and Use of Laboratory Animals ${ }^{2}$ (PHS Policy) allow an IACUC to carry out protocol reviews by either the full committee (FCR) or a designated member (DMR) ${ }^{3}$. An IACUC can use DMR for a protocol if the institution has a written policy that documents the assignment criteria and the procedures for processing the protocols. The DMR process must be in full compliance with all applicable requirements of the AWARs and PHS Policy and the designated reviewer must use the same criteria that are applicable to protocols undergoing FCR. DMR may be restricted to protocols involving noninvasive or acute procedures or may be used for continuing and triennial reviews with no or minor changes. In DMR, the designated member has the authority to approve a protocol, require modifications to secure approval or refer it to the full committee. The intent of DMR is to reduce the workload of the IACUC at convened meetings and to enable a faster protocol review and approval process. However, any member of an IACUC can ask for a FCR at any point during the DMR process. Thus, Liz Smith is right in saying that any member of the IACUC can refer the protocol for FCR if he or she has concerns about the protocol.

In this scenario, the AV may have been satisfied with the justification to withhold analgesics or may have unintentionally missed the potential concern. If the PI provided an appropriate justification to withhold analgesics, then the animals fall into USDA category E, and the PI must have considered and described alternatives to the proposed procedure in the protocol. In either case, Sean Smith's concern about the welfare of the animals used in the proposed research and the appropriate use of an analgesic postoperatively should be addressed via a FCR. As an IACUC member, Sean Smith should express his concern to the AV and IACUC chair (request for FCR) regarding the need for appropriate pain assessment and control on this protocol.

The Guide for the Care and Use of Laboratory Animals (the Guide) 4 expects the IACUC to use professional judgment when reviewing protocols with procedures that may cause more than momentary distress or pain and to take all necessary steps to alleviate or minimize pain and distress, unless scientifically justified. In addition, US Government Principle IV (ref. 5) states, "unless the contrary is established, investigators should consider that procedures 